\title{
Effect of short-term combined alkaline stress on antioxidant metabolism, photosynthesis, and leaf-air temperature difference in sorghum
}

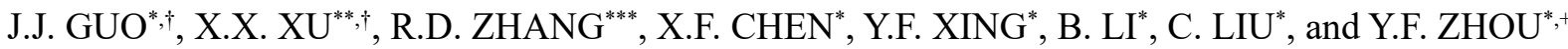 \\ College of Agronomy, Shenyang Agricultural University, Shenyang, Liaoning, China* \\ Tongliao Agricultural Technology Extension Station, Tongliao, Inner Mongolia, China** \\ Institute of Cash Crops, Shanxi Academy of Agricultural Sciences, Taiyuan, Shanxi, China***
}

\begin{abstract}
Alkaline stress is important abiotic stress that restricts the growth and physiological activity of sorghum (Sorghum bicolor L. Moench). We aimed to investigate the effects of alkaline stress on alkali-tolerant SX44B and alkali-sensitive 262B sorghum inbred lines. The results showed that alkaline stress decreased the content of chlorophyll, activity of photosystem II, net photosynthetic rate, and destroyed chloroplast morphology. These changes were less pronounced in SX44B, possibly owing to its higher antioxidant enzyme activity and nonphotochemical quenching. Alkaline stress decreased water content, transpiration rate, and stomatal conductance while increasing the leaf temperature, with the effect being more pronounced in 262B. A significant correlation was observed between leaf-air temperature difference $(\Delta \mathrm{T})$ and relative water content and gas-exchange parameters, especially in $262 \mathrm{~B}$. Therefore, $\Delta \mathrm{T}$ is an effective indicator for monitoring changes in sorghum leaves under alkaline stress and evaluating the alkali tolerance of different sorghum germplasm.
\end{abstract}

Keywords: alkaline stress; antioxidant enzymes; chlorophyll fluorescence; leaf-air temperature difference; physiology.

\section{Introduction}

Alkalization is a common ecological problem all over the world. Alkaline stress is one of the main abiotic stresses which can inhibit the growth and development of crops and is harmful for crop production. According to the statistics from FAO, the area of salt-alkali land in the world is about 954 million hectares and is increasing at a rate of 1-1.5 million hectares per year (Ren et al. 2017, Leng et al. 2020). The salt-alkali stress of plants mainly comes

\section{Highlights}

- The alkali-tolerant sorghum inbred line maintains high photosynthetic performance

- The alkali-tolerant line shows efficient antioxidant enzyme activity and nonphotochemical quenching

- A leaf-air temperature difference is an indicator of the alkaline tolerance of sorghum

\author{
Received 3 August 2021 \\ Accepted 10 December 2021 \\ Published online 8 February 2022 \\ ${ }^{+}$Corresponding author \\ e-mail: zhouyufei@syau.edu.cn
}

\footnotetext{
Abbreviations: APX - ascorbate peroxidase; CAT - catalase; Chl - chlorophyll; $C_{\mathrm{i}}$ - intercellular $\mathrm{CO}_{2}$ concentration; $\mathrm{CK}$ - no alkaline stress; $E$ - transpiration rate; $\mathrm{F}_{0}$ - minimal fluorescence yield of the dark-adapted state; $\mathrm{F}_{\mathrm{m}}-$ maximum fluorescence yield after 30-min dark adaptation; FM - fresh mass; $\mathrm{F}_{\mathrm{m}}{ }^{\prime}$ - instantaneous maximum fluorescence under illumination; $\mathrm{F}_{\mathrm{v}} / \mathrm{F}_{\mathrm{m}}-$ maximal quantum yield of PSII photochemistry; $g_{\mathrm{s}}$ - stomatal conductance; IL - different inbred lines; NPQ - nonphotochemical quenching; $P_{\mathrm{N}}-$ net photosynthetic rate; POD - peroxidase; ROS - reactive oxygen species; RWC - relative water content; SOD - superoxide dismutase; $\mathrm{TR}$ - different treatments; TS - alkaline stress; $\Delta \mathrm{T}$ - the difference between leaf temperature and ambient temperature; $\varepsilon$ - extinction coefficient; $\Phi_{\mathrm{PSII}}$ - effective quantum yield of PSII photochemistry.

Acknowledgments: This work is financially supported by the China Agriculture Research System of MOF and MARA (CARS-0613.5-A17).

${ }^{\dagger}$ These authors contributed equally to this work.

Conflict of interest: The authors declare that they have no conflict of interest.
} 
from the neutral salts $\mathrm{NaCl}$ and $\mathrm{Na}_{2} \mathrm{SO}_{4}$ and the alkaline salts $\mathrm{Na}_{2} \mathrm{CO}_{3}$ and $\mathrm{NaHCO}_{3}$ (Munns and Tester 2008). The effect of alkaline stress on crops is much greater than that of neutral salts because of its higher $\mathrm{pH}$ value (Amini et al. 2016). Sorghum [Sorghum bicolor (L.) Moench] is often planted on marginal land, which makes it vulnerable to alkaline stress. Sorghum researchers attempt to utilize alkali-tolerant germplasm to mitigate the effects of alkaline stress on its production (Huang 2018). Therefore, it is crucial to understand the physiological response of sorghum to alkaline stress and develop suitable evaluation methods.

Alkaline stress causes water deficit in plants, leading to physiological drought (Huang 2018). This causes the accumulation of large amounts of reactive oxygen species (ROS) in crops, resulting in oxidative stress (Sewelam et al. 2016, Choudhury et al. 2017), membrane lipid peroxidation, and damage to organelles (Noreen et al. 2009). To avoid the oxidative damage caused by ROS, the antioxidant system in crops undergoes a series of reactions to remove excess ROS. The enzymes that play a key role in this reaction include superoxide dismutase (SOD), peroxidase (POD), catalase (CAT), and ascorbate peroxidase (APX). When crops encounter alkaline stress, these enzymes improve the alkali-tolerant ability of plants by ensuring the prompt elimination of ROS. Hence, the plants can maintain normal growth and physiological and biochemical activities by effectively reducing the damage caused by ROS (Tuna et al. 2008). Under alkaline stress, the CAT synthesis pathway is inhibited, while there is an increase in the activities of POD and glutathione peroxidase (GPX). However, an increase in stress intensity leads to a decrease in the activities of SOD, APX, and CAT (Zhao et al. 2016). Alkaline stress elicits varied responses from different antioxidant enzymes. Thus, there is a lot of interest in the key enzymes which play a critical role in scavenging ROS from damaged organelles in sorghum.

The most prominent effect of alkaline stress is apparently on photosynthesis. Alkaline stress increases the activity of chlorophyll-degrading enzymes, resulting in a decrease in chlorophyll content (Yang et al. 2011). Additionally, alkaline stress can also damage the ultrastructure of chloroplasts and cause dissociation of the thylakoid membrane by peroxidation (Wang et al. 2010). This is followed by inhibition of the activity of PSI and PSII and, finally, decreased photosynthesis in plants (Yang et al. 2009, Qiao et al. 2013). With the increase in alkaline stress, the photosynthetic capacity of crops declines from stomatal restriction to nonstomatal restriction, both of which cause a decrease in stomatal conductance $\left(g_{\mathrm{s}}\right)$ and transpiration rate $(E)$ (Liu 2010). The reduction of $E$ causes slow dissipation of heat in the leaf, leading to an increase in leaf temperature. Therefore, changes in leaf temperature can be used as an effective indicator for evaluating abiotic stress levels (Zhang et al. 2019a). Blum et al. (1982) demonstrated that the leaf temperature is related to the leaf water potential. Leaf-air temperature difference $(\Delta \mathrm{T})$ (the difference between leaf temperature and ambient temperature) can reflect the leaf moisture status more accurately than leaf temperature alone. Zhang et al. (2019a) also concluded that the $\Delta \mathrm{T}$ can reflect the moisture status of sorghum leaves and can be used to identify the waterlogging tolerance conditions of sorghum. However, at present, there are very few studies on the effect of short-term alkaline stress on photosynthetic characteristics, in terms of the activities of PSI and PSII, and changes in the leaf temperature of sorghum. In particular, the relationship between $\Delta \mathrm{T}$ and the changes in photosynthesis and water status is still unclear.

Therefore, the objectives of this research were to (1) explore the changes in key enzymes of antioxidant metabolism, photosynthetic characteristics, and leaf temperature in different alkali-tolerant sorghum varieties under short-term combined alkaline stress, (2) identify the relationship between $\Delta \mathrm{T}$ and changes in photosynthesis and leaf water content. These results may be used to develop a functional approach to promote the performance of sorghum under alkaline stress. It will aid in the development of a novel method for monitoring alkalitolerant genotypes for future applications.

\section{Materials and methods}

Plant materials and experimental design: The experiments were carried out in the physiological laboratory of sorghum in Shenyang Agricultural University, using the method of indoor culture. Two sorghum inbred lines, SX44B and 262B, were used in this study. Based on our previous study results, SX44B exhibits tolerance to alkali stress, whereas $262 \mathrm{~B}$ is sensitive to alkali stress. Similarsized sorghum seeds of each of the inbred lines were selected for germination. The seeds were disinfected with sodium hypochlorite $(10 \%)$ solution for 5-10 $\mathrm{min}$ and washed with distilled water. Thereafter, they were placed on a moist filter paper in a Petri dish, before being kept in an incubator for culture. The settings of the incubator were maintained at $28 / 25^{\circ} \mathrm{C}$ day/night, with a $12-\mathrm{h}$ photoperiod and light intensity of $280 \mu \mathrm{mol}$ (photon) $\mathrm{m}^{-2} \mathrm{~s}^{-1}$. Three days later, 20 well-developing seedlings were selected and inserted into a PCR plate with the bottom cut off. The plate was placed in a square basin covered with black shading paper outside. The culture medium was changed to $1 / 2$ Hoagland's nutrient solution after $4 \mathrm{~d}$ of distilled water treatment. After $3 \mathrm{~d}$ of nutrient solution treatment, the alkaline stress was induced with $75 \mathrm{mmol} \mathrm{L}^{-1}$ solution of $\mathrm{NaHCO}_{3}$ and $\mathrm{Na}_{2} \mathrm{CO}_{3}$ (molar ratio 9:1, pH = 8.9). One-half strength Hoagland's nutrient solution was used as control. After $3 \mathrm{~d}$ of stress treatment, the related indexes were measured.

Morphological parameters: From each treatment group, plants of similar growth status were selected for morphological index measurement. The lengths and fresh mass of shoots and roots were measured using a ruler and a onethousandth electronic balance, respectively. The dry mass of shoots and roots was measured after the samples were dried in an $80^{\circ} \mathrm{C}$ oven until no significant reduction in the 
sample mass was observed. Each treatment was repeated five times.

Superoxide dismutase (SOD, EC 1.15.1.1) activity: For the measurement of SOD activity, $0.3 \mathrm{~g}$ of fresh leaves or roots were ground in a mortar, along with $9 \mathrm{~mL}$ of $50 \mathrm{mmol} \mathrm{L}^{-1}$ phosphoric acid buffer ( $\mathrm{pH} \mathrm{7.8)} \mathrm{and} \mathrm{a} \mathrm{small}$ amount of quartz sand, until complete homogenization was achieved. The solution was transferred into a $10-\mathrm{mL}$ centrifuge tube and centrifuged at $12,000 \times g$ for $15 \mathrm{~min}$. The supernatant was treated as the crude extract of the enzyme. Subsequently, $1.5 \mathrm{~mL}$ of phosphate buffer, $0.3 \mathrm{~mL}$ of $130 \mathrm{mmol} \mathrm{L}^{-1}$ methionine (Met) solution, $0.3 \mathrm{~mL}$ of $750 \mu \mathrm{mol} \mathrm{L} \mathrm{L}^{-1}$ nitroblue tetrazolium solution, $0.3 \mathrm{~mL}$ of $100 \mu \mathrm{mol} \mathrm{L} \mathrm{L}^{-1}$ ethylenediamine-tetraacetic acid disodium salt solution, $0.3 \mathrm{~mL}$ of $20 \mu \mathrm{mol} \mathrm{L} \mathrm{L}^{-1}$ riboflavin solution, $0.25 \mathrm{~mL}$ of distilled water, and $0.05 \mathrm{~mL}$ of enzyme solution were added into a beaker in turns. The two control groups had the same amount of distilled water added to them, instead of the enzyme solution. Additionally, one control was kept in a dark environment, while the other was exposed to light. The light environment was set up with a light intensity of $250 \mu \mathrm{mol}$ (photon) $\mathrm{m}^{-2} \mathrm{~s}^{-1}$ and a duration of $20 \mathrm{~min}$. Thereafter, all samples were moved to the dark to stop the reaction. The absorbance value of each treatment was measured at $560 \mathrm{~nm}$ (UV-VIS spectrophotometer Hitachi U-1800, Tokyo, Japan), with the shading tube serving as the blank (Yin et al. 2009). SOD total activity [U g $\left.\mathrm{g}^{-1}(\mathrm{FM})\right]=$ $[($ Ack $-\mathrm{AE}) \mathrm{VT}] /(0.5 \mathrm{Ack} \times \mathrm{M} \times \mathrm{VS})$. Ack - the absorbance of the illuminated control tube; AE - the absorbance of a sample tube; VT - total volume of enzyme solution $[\mathrm{mL}]$; $\mathrm{VS}$ - the dosage of enzyme solution during measurement [mL]; M - sample fresh mass [g].

Peroxidase (POD, EC 1.11.1.7) activity: The crude enzyme solution was prepared as mentioned earlier. The reaction system was prepared as described below. $140 \mu \mathrm{L}$ of $50 \mathrm{mmol} \mathrm{L}^{-1}$ guaiacol solution were added into $250 \mathrm{~mL}$ of $50 \mathrm{mmol} \mathrm{L}^{-1}$ phosphate buffer $(\mathrm{pH}=6.0)$. After complete dissolution, $95 \mu \mathrm{L}$ of $30 \% \mathrm{H}_{2} \mathrm{O}_{2}$ solution was added and thoroughly mixed. The mixture solution, prepared by mixing $30 \mu \mathrm{L}$ of enzyme solution and $3 \mathrm{~mL}$ of reaction system, was spectrophotometrically determined at $470 \mathrm{~nm}$ (UV-VIS spectrophotometer Hitachi U-1800, Tokyo, Japan) (Fielding and Hall 1978). The measurement was repeated four times, with a time interval of $1 \mathrm{~min}$ between the repeats. The activity of POD was calculated using the extinction coefficient $(\varepsilon)$ of $26.6 \mathrm{mM}^{-1} \mathrm{~cm}^{-1}$. $\left.\mathrm{POD}[\mu \mathrm{mol} \mathrm{g}-1 \mathrm{FM}) \mathrm{min}^{-1}\right]=\left(\Delta \mathrm{A}_{470} \times \mathrm{Vt}\right) /(\varepsilon \times \mathrm{d} \times \mathrm{M} \times$ $\mathrm{Vs} \times \mathrm{t}) . \Delta \mathrm{A}_{470}-$ change in absorbance within $1 \mathrm{~min}$; $\mathrm{M}$ - sample fresh mass [g]; $\mathrm{t}$ - reaction time [min]; $\mathrm{Vt}$ - total volume of extracted enzyme solution $[\mathrm{mL}]$; Vs - enzyme solution volume of samples during measurement $[\mathrm{mL}] ; \varepsilon-$ extinction coefficient $\left[\mathrm{mM}^{-1} \mathrm{~cm}^{-1}\right]$; $\mathrm{d}$ - cuvette thickness $[\mathrm{cm}]$.

Catalase (CAT, EC 1.11.1.6) activity: The crude enzyme solution was prepared as mentioned earlier. The reaction system was prepared as described below. $250 \mu \mathrm{l}$ of
$50 \mathrm{mmol} \mathrm{L}^{-1}$ phosphate buffer $(\mathrm{pH}=7.0)$ were added into $386.5 \mu \mathrm{L}$ of $30 \% \mathrm{H}_{2} \mathrm{O}_{2}$, and the solution was thoroughly mixed. The mixture solution, prepared by mixing $0.1 \mathrm{~mL}$ of enzyme solution and $3 \mathrm{~mL}$ of the reaction system, was spectrophotometrically determined at $240 \mathrm{~nm}$ (UV-VIS spectrophotometer Hitachi U-1800, Tokyo, Japan) (Yordanova et al. 2004). The measurement was repeated four times, with an interval of 1 min between the repeats. The activity of CAT was calculated using the extinction coefficient of $43.6 \mathrm{mM}^{-1} \mathrm{~cm}^{-1}$. CAT $\left[\mu \mathrm{mol} \mathrm{g}^{-1}(\mathrm{FM}) \mathrm{min}^{-1}\right]=\left(\Delta \mathrm{A}_{240} \times \mathrm{Vt}\right) /(\varepsilon \times \mathrm{d} \times \mathrm{M} \times \mathrm{Vs} \times \mathrm{t})$. $\Delta \mathrm{A}_{240}$ - change in absorbance within $1 \mathrm{~min} ; \mathrm{M}$ - sample fresh mass $[\mathrm{g}] ; \mathrm{t}$ - reaction time [min]; $\mathrm{Vt}$ - total volume of extracted enzyme solution [mL]; Vs - enzyme solution volume of samples during measurement $[\mathrm{mL}] ; \varepsilon$ - extinction coefficient $\left[\mathrm{mM}^{-1} \mathrm{~cm}^{-1}\right] ; \mathrm{d}$ - cuvette thickness $[\mathrm{cm}]$.

Ascorbate peroxidase (APX, EC 1.11.1.11) activity: The crude enzyme solution was prepared as same as described earlier. The reaction system consisted of $1.8 \mathrm{~mL}$ of $50 \mathrm{mmol} \mathrm{L}^{-1}$ phosphate buffer $(\mathrm{pH}=7), 0.1 \mathrm{~mL}$ of $15 \mathrm{mmol} \mathrm{L}^{-1}$ ascorbic acid, $1 \mathrm{~mL}$ of $0.3 \mathrm{mmol} \mathrm{L} \mathrm{m}^{-1} \mathrm{H}_{2} \mathrm{O}_{2}$, and $0.1 \mathrm{~mL}$ of enzyme solution. The control consisted of all the solutions mentioned above, except for the enzyme solution (which was replaced by phosphate buffer). The control was defined as zero, and the absorbance was measured at $\mathrm{OD}_{290}$ (Yordanova et al. 2004). The measurement was repeated four times, with an interval of $1 \mathrm{~min}$ between the repeats. The activity of APX was calculated using the extinction coefficient of $2.8 \mathrm{mM}^{-1} \mathrm{~cm}^{-1}$. APX $\left[\mu \mathrm{mol} \mathrm{g}^{-1}(\mathrm{FM}) \mathrm{min}^{-1}\right]=\left(\Delta \mathrm{A}_{290} \times \mathrm{V} 1\right) /(\varepsilon \times \mathrm{d} \times \mathrm{V} 2 \times \mathrm{t} \times \mathrm{M})$. $\Delta \mathrm{A}_{290}$ - change in absorbance within $1 \mathrm{~min} ; \mathrm{V} 1$ - total volume of extracted enzyme solution [mL]; V2 - enzyme solution volume for measurement $[\mathrm{mL}] ; \mathrm{t}$ - reaction time [min]; M-sample fresh mass [g]; $\varepsilon$ - extinction coefficient $\left[\mathrm{mM}^{-1} \mathrm{~cm}^{-1}\right] ; \mathrm{d}$ - cuvette thickness $[\mathrm{cm}]$.

Chlorophyll (Chl) content: Fresh sorghum leaves (0.3 g) were cut into pieces and put into a $10-\mathrm{mL}$ centrifuge tube. Five milliliters of anhydrous ethanol and $5 \mathrm{~mL}$ of acetone were added into the centrifuge tube and extracted in dark for $48 \mathrm{~h}$. The solution was spectrophotometrically determined at $652 \mathrm{~nm}$ (a mixture of ethanol and acetone solution served as blank) (UV-VIS spectrophotometer Hitachi U-1800, Tokyo, Japan) (Wintermans and De Mots 1965). Total Chl $\left[\mathrm{mg} \mathrm{g}^{-1}\right]=\left(\mathrm{A}_{652} / 34.5\right) \times(\mathrm{V} / \mathrm{M}) \times 1,000$. $\mathrm{A}_{652}$ - absorbance at $652 \mathrm{~nm} ; \mathrm{V}$ - total volume of extract $[\mathrm{mL}] ; \mathrm{M}-$ sample fresh mass $[\mathrm{g}]$.

Chloroplast ultrastructure: A leaf piece $(1 \times 3 \mathrm{~mm})$ was cut from the middle part of sampled leaves in each treatment with a sharp blade, avoiding the central vein. Then the leaf pieces were placed in a glass bottle containing $2.5 \%$ pentanediol. The bottle was degassed to allow the pieces completely immerse in the pentanediol solution and stored in a refrigerator at $4^{\circ} \mathrm{C}$. Each fixed sample was rinsed three times with phosphoric acid buffer $(\mathrm{pH} \mathrm{7.8)}$ and then fixed in 1\% osmic acid for $2 \mathrm{~h}$. Then the samples were dehydrated by 50 and $70 \%$ ethanol, 80 and $90 \%$ acetone (15 min each treatment), and finally 
treated with $100 \%$ acetone three times ( 30 min each time). The samples were immersed in a mixture of propylene oxide and $S P O N-812$ and placed in an incubator for 12-h polymerization. Sections were cut with Leica EM UC7 ultra-thin microtome (Wetzlar, Germany) and then stained with uranyl acetate and lead citrate. Samples were observed with an LSM500 transmission electron microscope (Zeiss, Germany).

Chl fluorescence parameters: The second top leaves of uniform sorghum seedling were selected for the measurement. The leaf samples were cleaned up and darkadapted for $30 \mathrm{~min}$ at room temperature before analysis. FluorCam FC 800-O/2020 chlorophyll fluorometer (Brno, Czech Republic) was used to take photos of the leaves. The parameters to capture chlorophyll fluorescence transients in a batch of images, including shutter, sensitivity, super, far, and act, were set to $1,25 \%, 60 \%$, $50 \%$ and $30 \%$, respectively. The minimal fluorescence $\left(\mathrm{F}_{0}\right)$ was measured under very low light $[<0.1 \mu \mathrm{mol}$ (photon) $\left.\mathrm{m}^{-2} \mathrm{~s}^{-1}\right]$. The maximal fluorescence $\left(\mathrm{F}_{\mathrm{m}}\right)$ was measured under a saturation pulse $\left[10,000 \mu \operatorname{mol}\left(\right.\right.$ photon) $\mathrm{m}^{-2} \mathrm{~s}^{-1}$, a pulse time of $0.7 \mathrm{~s}$ ]. The maximal fluorescence of the light-adapted state $\left(\mathrm{F}_{\mathrm{m}}^{\prime}\right)$ was measured after the leaf was adapted to actinic light $\left[800 \mu \mathrm{mol}\left(\right.\right.$ photon) $\mathrm{m}^{-2} \mathrm{~s}^{-1}$ ] for $15 \mathrm{~min}$ (Zhang et al. 2019b). The software FluorCam was used for picture and data analyses (Hussain et al. 2019). $F_{v} / F_{m}$ (photochemical efficiency of PSII) $=\left(F_{m}-\right.$ $\left.\mathrm{F}_{0}\right) / \mathrm{F}_{\mathrm{m}}$. NPQ (nonphotochemical quenching) $=\left(\mathrm{F}_{\mathrm{m}}-\mathrm{F}_{\mathrm{m}}{ }^{\prime}\right) /$ $\mathrm{F}_{\mathrm{m}}{ }^{\prime} \cdot \mathrm{F}_{0}$ - minimal fluorescence yield of the dark-adapted state; $F_{m}-$ maximum fluorescence yield after 30-min dark adaptation; $\mathrm{F}_{\mathrm{m}}{ }^{\prime}$ - instantaneous maximum fluorescence under illumination.

Photosynthetic parameters: The second top leaf of sorghum seedling was selected for the measurement. The photosynthetic index was measured under the light in the incubator. The net photosynthetic rate $\left(P_{\mathrm{N}}\right)$, stomatal conductance $\left(g_{\mathrm{s}}\right)$, transpiration rate $(E)$, and intercellular $\mathrm{CO}_{2}$ concentration $\left(C_{\mathrm{i}}\right)$ were measured using LI-6400 photosynthetic apparatus ( $L I-C O R, \mathrm{USA})$. Each treatment was repeated five times. The measuring light intensity was $1,000 \mu \mathrm{mol}$ (photon) $\mathrm{m}^{-2} \mathrm{~s}^{-1}$. The $\mathrm{CO}_{2}$ concentration was $385 \pm 5 \mu \mathrm{mol} \mathrm{mol}{ }^{-1}$ while the temperature was $28^{\circ} \mathrm{C}$. The measured leaf compartment area was $1 \times 2 \mathrm{~cm}$.

Leaf moisture: The fresh mass $\left(\mathrm{M}_{\mathrm{f}}\right)$ and dry mass $\left(\mathrm{M}_{\mathrm{d}}\right)$ of samples were measured. After measuring the fresh mass, the sample was immersed in distilled water for several hours. The samples were dried before being weighed. The immersion step was repeated until the weighing results were roughly equal to the previous one. The final result was considered as the saturated fresh mass $\left(\mathrm{M}_{\mathrm{t}}\right)$. $\operatorname{RWC}[\%]=\left(\mathrm{M}_{\mathrm{f}}-\mathrm{M}_{\mathrm{d}}\right) /\left(\mathrm{M}_{\mathrm{t}}-\mathrm{M}_{\mathrm{d}}\right) \times 100$.

Thermal infrared image acquisition: After $3 \mathrm{~d}$ of alkaline stress treatment, the CA1899 thermal infrared camera (CHAUVIN ARNOUX, France) was placed at one meter in front of the plants, to obtain infrared images and record the current air temperature $\left(\mathrm{T}_{\mathrm{a}}\right)$. After the infrared image was collected, the CA Infrared Analysis software was used to record and analyze the leaf temperature.

The measurement of the leaf temperature $\left(T_{1}\right)$ was performed at a distance of $2 \mathrm{~cm}$ from the tip of the uppermost leaf. Each measurement was performed on five plants, and the average was taken as $T_{1}$. The leaf temperature difference calculation formula was as follows: $\Delta \mathrm{T}=\mathrm{T}_{1}-\mathrm{T}_{\mathrm{a}}$.

Statistical analysis: Both one-way and two-way analysis of variance $(A N O V A)$, regression analysis, and Duncan's multiple range test were performed with DPS software (http://www.dpsw.cn/index.html). The results are expressed as the mean $\pm \mathrm{SD}$. The data were collated and plotted using GraphPad Prism8 software.

\section{Results}

Morphological parameters: Alkaline stress affected the growth of sorghum seedlings. As shown in Fig. 1S (supplement), alkaline stress had different effects on the two sorghum inbred lines. The leaves of alkali-sensitive inbred line 262B appeared withered and wilted, whereas the leaves of alkali-tolerant inbred line SX44B maintained a good appearance.

As shown in Fig. 1A, under alkaline stress, the shoot length of the two inbred lines showed a downward trend compared to that of the control. Among them, the decline of $262 \mathrm{~B}$ was greater than that of SX44B. The shoot length of SX44B and 262B decreased by 32.9 and $49.4 \%$, respectively, compared with that of the control. The difference in shoot length between the treatments of the two inbred lines was significant. Furthermore, significant interactions between inbred lines and alkaline stress were observed in the shoot length.

Under alkaline stress, the fresh mass of the shoot decreased in both inbred lines. The decrease of 262B was greater than that of SX44B. The fresh mass of shoot of 262B showed a significant decrease of $71.1 \%$ compared with that of the control. Additionally, there was a significant drop in the fresh mass of shoot of SX44B by $47.8 \%$. After stress, the shoot fresh mass of SX44B was higher than that of 262B; however, the result was not statistically significant (Fig. 1B). Also, there was a marked interaction between inbred lines and alkaline stress in the fresh mass of the shoot.

The data of shoot dry mass showed the same downward trend as that of shoot fresh mass under alkaline stress. The decline of shoot dry mass of 262B was more obvious than that of SX44B. Specifically, the shoot dry mass of $262 \mathrm{~B}$ decreased by $32.7 \%$, and that of SX44B decreased by $24.5 \%$. There were significant differences between the shoot dry mass of the two inbred lines and the corresponding control. After exposure to stress, there was no significant difference between the shoot dry mass of 262B and SX44B (Fig. 1C). However, no significant interactions between inbred lines and alkaline stress were observed in shoot dry mass. 


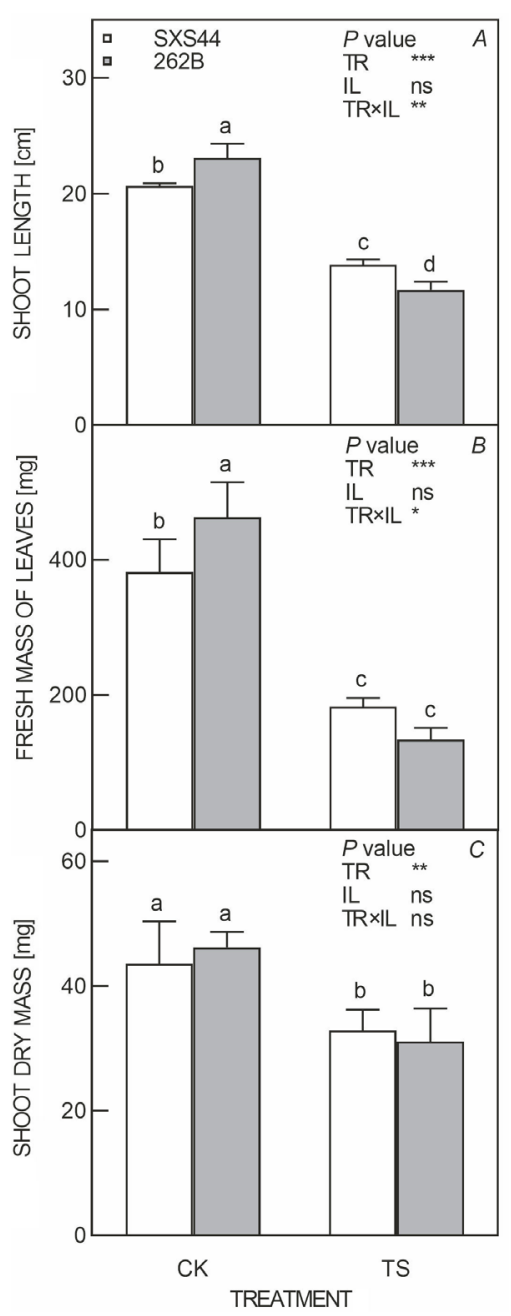

Fig. 1. Effect of alkaline stress on the agronomic traits of sorghum seedlings. $(A)$ shoot length; $(B)$ fresh mass of leaves; $(C)$ dry mass of shoot. TR - different treatments; IL - different inbred lines; CK - no alkaline stress; TS - alkaline stress. Significant differences $(P<0.05)$ were determined by different lowercase letters according to the one-way or two-way ANOVA followed by the least significant difference test. * - significant at the $P=0.05$ level. ** - significant at the $P=0.01$ level. *** - significant at the $P=0.001$ level. ns - no significant difference.

Antioxidant enzyme activities: The SOD activity showed an upward trend in both inbred lines under alkaline stress (Fig. 2A). The leaf SOD activity of SX44B and 262B increased by 47.8 and $40.1 \%$, respectively, compared to that of the control. The difference in SOD activity between the two treatments and each control was not significant and there was no significant difference in SOD activity between the two treatments.

After stress treatment, the POD activity in sorghum leaves increased in both inbred lines (Fig. 2B). The POD activity in SX44B and 262B leaves significantly increased by 125.7 and $82.9 \%$, respectively, when compared with that of the control. The increase of the POD activity in SX44B was higher than that of 262B, and the difference in
POD activity between the two treatments was statistically significant.

Under alkaline stress, the CAT activity of SX44B leaves significantly increased by $41.2 \%$ when compared to that of the control. There was a $14.7 \%$ increase in the CAT activity of 262B leaves compared with that of the control; however, it was not statistically significant. Although the CAT activity of SX44B leaves was higher than that of 262B, the difference was not significant (Fig. 2C).

After alkaline stress treatment, the APX activities of SX44B and 262B significantly increased by 88.7 and $42.5 \%$, respectively, when compared with that of the control. The APX activity of SX44B leaves was higher than that of 262B; however, the difference was not significant (Fig. 2D).

Thus, under alkaline stress, the antioxidant enzyme activities in SX44B were higher than those in 262B. Also, significant interactions between inbred lines and alkaline stress were observed in the activity of POD and APX, except for the activity of SOD and CAT. It showed that alkali-tolerant inbred lines can resist the damaging effects of alkaline stress in plants by improving the activity of antioxidant enzymes. It can remove the ROS in the plants effectively and reduce the degree of membrane lipid peroxidation, leading to better alkaline resistance in plants.

Chl content and chloroplast ultrastructure: $\mathrm{Chl}$ is the main pigment for photosynthesis in plants. Under the effect of $75 \mathrm{mmol} \mathrm{L}^{-1}$ mixed alkaline stress (Fig. 3), the Chl content of SX44B and 262B showed a significant decrease of 41.2 and $66.4 \%$, respectively, when compared with that of the control. Additionally, the Chl content of SX44B was significantly higher than that of 262B. But, no significant interactions between inbred lines and alkaline stress were found in Chl content.

The chloroplast had a complete structure and a full oval shape. Its double-membrane structure was visible. A small number of osmiophilic granules was found in the chloroplasts. The thylakoid sheets in the chloroplasts were stacked neatly (Fig. 2S $A-D$, supplement). Under alkaline stress, the chloroplast of SX44B suffered less damage, while still maintaining a normal oval shape. The chloroplast double-layer membrane structure was almost completely intact. The thylakoid sheets in the chloroplast were arranged loosely, twisted, and swollen. However, some of the layers showed a better arrangement. By contrast, under saline stress, the chloroplast of 262B deformed from the original long ellipse to a spherical shape. The chloroplast membrane was completely dissolved. The thylakoid lamella disintegrated and disappeared (Fig. 2SE-H, supplement).

Chl fluorescence parameters: Under saline stress, the $\mathrm{F}_{0}$ decreased in both seedlings of these two sorghum inbred lines (Fig. 4). The $\mathrm{F}_{0}$ of SX44B and 262B decreased by 0.3 and $15.9 \%$, respectively. The value shown by $262 \mathrm{~B}$ was significantly different compared with that of control, while that of SX44B was not. The $\mathrm{F}_{0}$ of SX44B was 1.23 times higher than that of $262 \mathrm{~B}$, and the difference in $\mathrm{F}_{0}$ between the two inbred lines was statistically significant. 


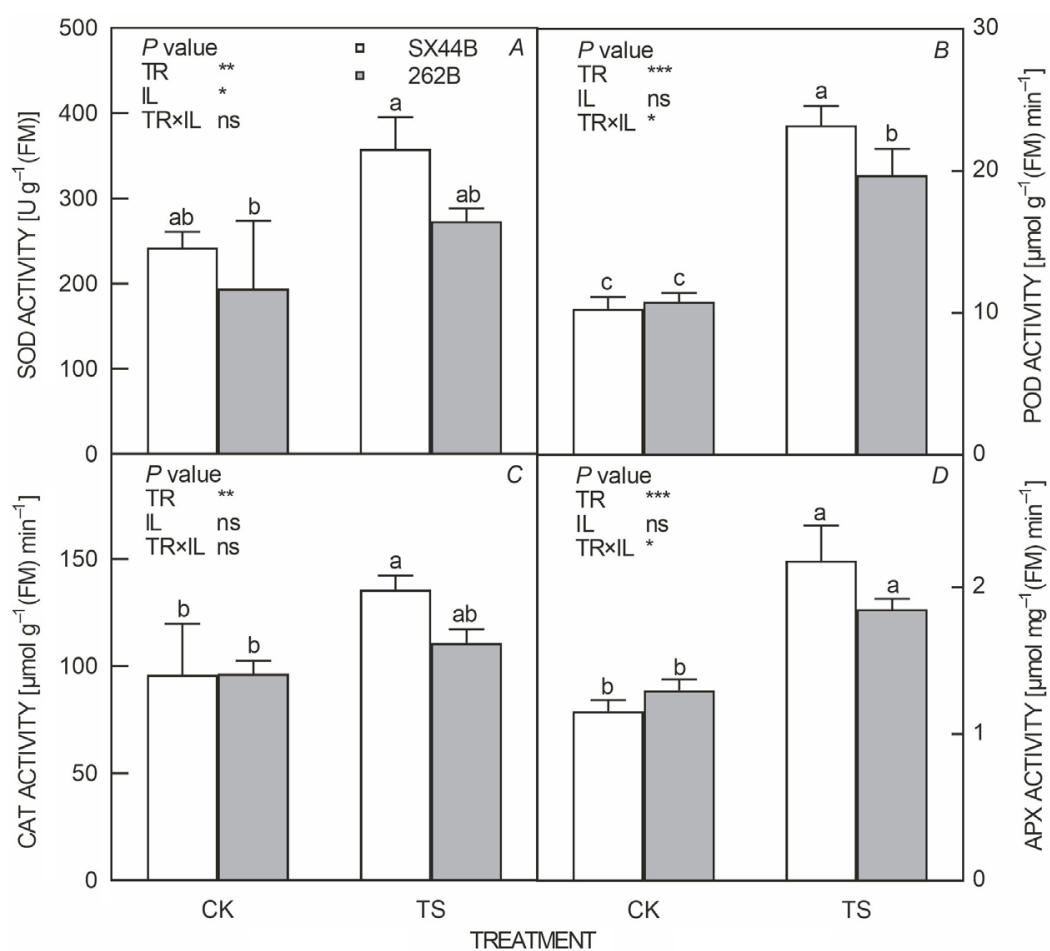

Fig. 2. Effect of alkaline stress on antioxidant enzyme activity in leaves of sorghum seedlings. (A) superoxide dismutase (SOD) activity; (B) peroxidase (POD) activity; (C) catalase (CAT) activity; (D) ascorbate peroxidase (APX) activity. TR - different treatments; IL - different inbred lines; FM fresh mass; CK - no alkaline stress; TS alkaline stress. Significant differences $(P<0.05)$ were determined by different lowercase letters according to the one-way or two-way ANOVA followed by the least significant difference test. ${ }^{*}$ - significant at the $P=0.05$ level. ** - significant at the $P=0.01$ level. *** - significant at the $P=0.001$ level. ns - no significant difference.

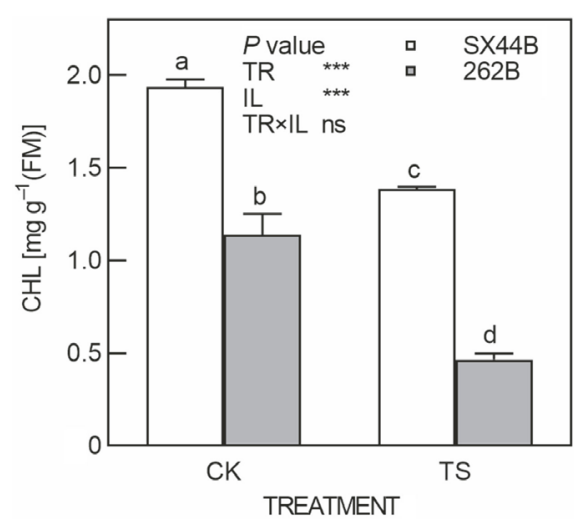

Fig. 3. Effect of alkaline stress on chlorophyll content in sorghum seedlings. Chl - chlorophyll; TR - different treatments; IL - different inbred lines; FM - fresh mass; CK - no alkaline stress; TS - alkaline stress. Significant differences $(P<0.05)$ were determined by different lowercase letters according to the one-way or two-way ANOVA followed by the least significant difference test. * - significant at the $P=0.05$ level. ** - significant at the $P=0.01$ level. *** - significant at the $P=0.001$ level. ns - no significant difference.

There was an 11.2 and $21.8 \%$ drop in $\mathrm{F}_{\mathrm{v}} / \mathrm{F}_{\mathrm{m}}$ in SX44B and $262 \mathrm{~B}$, respectively. The value of $\mathrm{F}_{\mathrm{v}} / \mathrm{F}_{\mathrm{m}}$ in SX44B was not significantly different compared to that of the control, while that of 262B showed a significant difference. The $F_{v} / F_{m}$ of SX44B was higher than that of $262 B$, and there was a significant difference in $\mathrm{F}_{\mathrm{v}} / \mathrm{F}_{\mathrm{m}}$ between the two inbred lines. Thus, this implies that $262 \mathrm{~B}$ was more easily affected by alkaline stress than SX44B.
Under alkaline stress, the NPQ of SX44B showed a significant increase of $96.3 \%$ compared to that of the control, while that of $262 \mathrm{~B}$ was $62.4 \%$ lower than that of the control, with the result not being significant. The NPQ of SX44B was 2.9 times higher than that of $262 \mathrm{~B}$, and the difference was statistically significant.

Under alkaline stress, the $\Phi_{\text {PSII }}$ of SX44B and 262B showed a significant decrease of 54.3 and $79.4 \%$, respectively, compared to that of the control. The $\Phi_{\text {PSII }}$ of SX44B was 2.3 times higher than that of $262 \mathrm{~B}$, which was significant.

Furthermore, there was a marked interaction between inbred lines and alkaline stress across all $\mathrm{Chl}$ fluorescence parameters.

Photosynthetic parameters: After alkaline stress treatment, the $P_{\mathrm{N}}$ of SX44B and 262B significantly decreased by 34.9 and $55.0 \%$, respectively, compared to that of the control (Fig. 5A). Alkaline stress caused the $g_{\mathrm{s}}$ of SX44B and $262 \mathrm{~B}$ to significantly decrease by 39.0 and $64.8 \%$, respectively, compared to that of the control. The decline of 262B was higher than that of SX44B. The $g_{\mathrm{s}}$ of SX44B was 2.0 times higher than that of $262 \mathrm{~B}$, with a significant difference (Fig. $5 B$ ). After the alkaline treatment, the $C_{\mathrm{i}}$ of SX44B and 262B significantly increased by 25.0 and $162.0 \%$, respectively, compared with that of the control. After stress, the $C_{\mathrm{i}}$ of $262 \mathrm{~B}$ was 2.0 times higher than that of SX44B, with a significant difference (Fig. 5C). The trend of $E$ was similar to that of $P_{\mathrm{N}}$ and $g_{\mathrm{s}}$. Under alkaline stress, the $E$ of the two sorghum inbred lines decreased significantly. The decline of SX44B was lower than that of 262B. The $E$ of SX44B was 1.5 times higher than that of 262B, with a significant difference (Fig. 5D). 


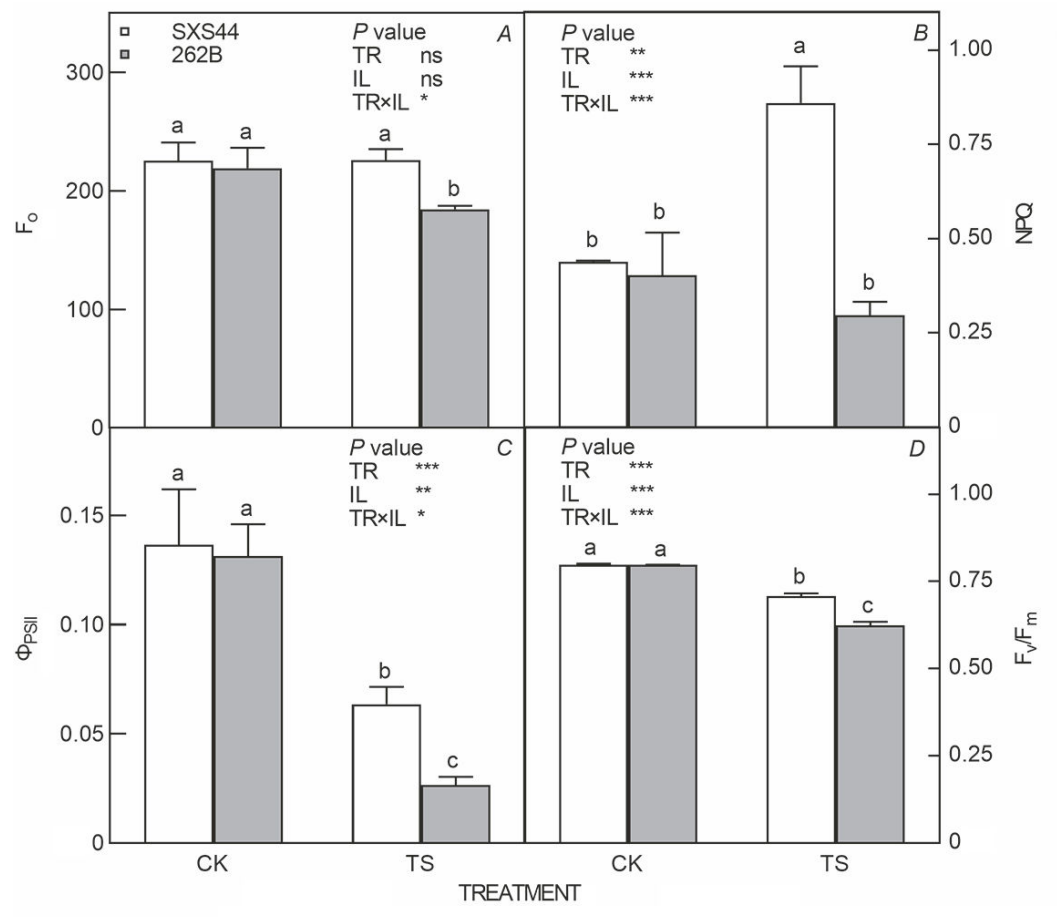

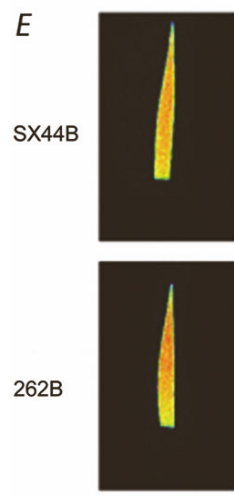

CK-Fo
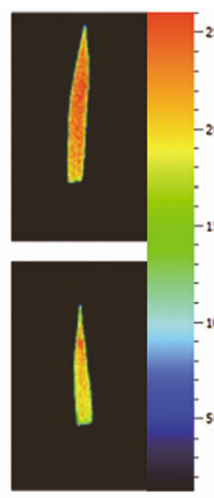

TS-F。
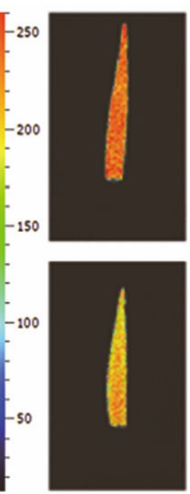

CK-Fv/Fm

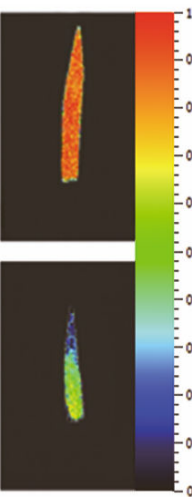

TS-Fv/Fm

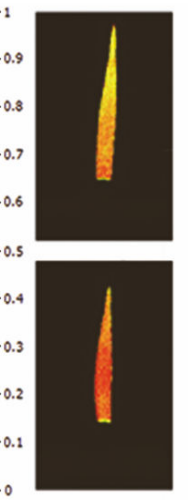

CK-NPQ

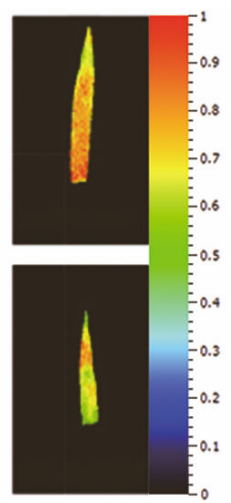

TS-NPQ

Fig. 4. Effect of alkaline stress on Chl fluorescence parameters of sorghum seedlings. $(A)$ and $(E)$ minimal fluorescence $\left(\mathrm{F}_{0}\right) ;(B)$ and $(E)$ nonphotochemical quenching (NPQ); $(C)$ effective quantum yield of PSII photochemistry $\left(\Phi_{\mathrm{PSII}}\right) ;(D)$ and $(E)$ maximum photochemical efficiency of PSII $\left(\mathrm{F}_{\mathrm{v}} / \mathrm{F}_{\mathrm{m}}\right)$. TR - different treatments; IL - different inbred lines; CK - no alkaline stress; TS - alkaline stress. Significant differences $(P<0.05)$ were determined by different lowercase letters according to the one-way or two-way ANOVA followed by the least significant difference test. * - significant at the $P=0.05$ level. ** - significant at the $P=0.01$ level. *** - significant at the $P=0.001$ level. ns - no significant difference.

Taken together, significant interactions between inbred lines and alkaline stress were found in $P_{\mathrm{N}}, g_{\mathrm{s}}$, and $C_{\mathrm{i}}$, except for $E$.

RWC and leaf temperature: It can be seen from Fig. 6 that alkaline stress caused physiological drought and dehydration in seedlings. There were no significant differences in the RWC of the leaves in the two sorghum inbred lines in the control group. Under alkaline stress, the RWC of the leaves in the two sorghum inbred lines decreased. The decline of 262B was higher than that of SX44B, and the values were significantly different from that of the control. However, the RWC of leaves decreased slightly after stress in SX44B and was not significantly different compared with that of the control. There was no notable interaction between inbred lines and alkaline stress in RWC. The effect of alkaline stress on the leaf temperature difference $(\Delta T)$ of sorghum leaves is shown in Fig. 7. The average $\Delta \mathrm{T}$ of SX44B and 262B increased significantly by 0.3 and $1.70^{\circ} \mathrm{C}$, respectively, compared with that of the control. The increasing trend of $\Delta \mathrm{T}$ was more obvious in the alkali-sensitive variety $262 \mathrm{~B}$. Moreover, there was a significant interaction between inbred lines and alkaline stress in $\Delta \mathrm{T}$. 


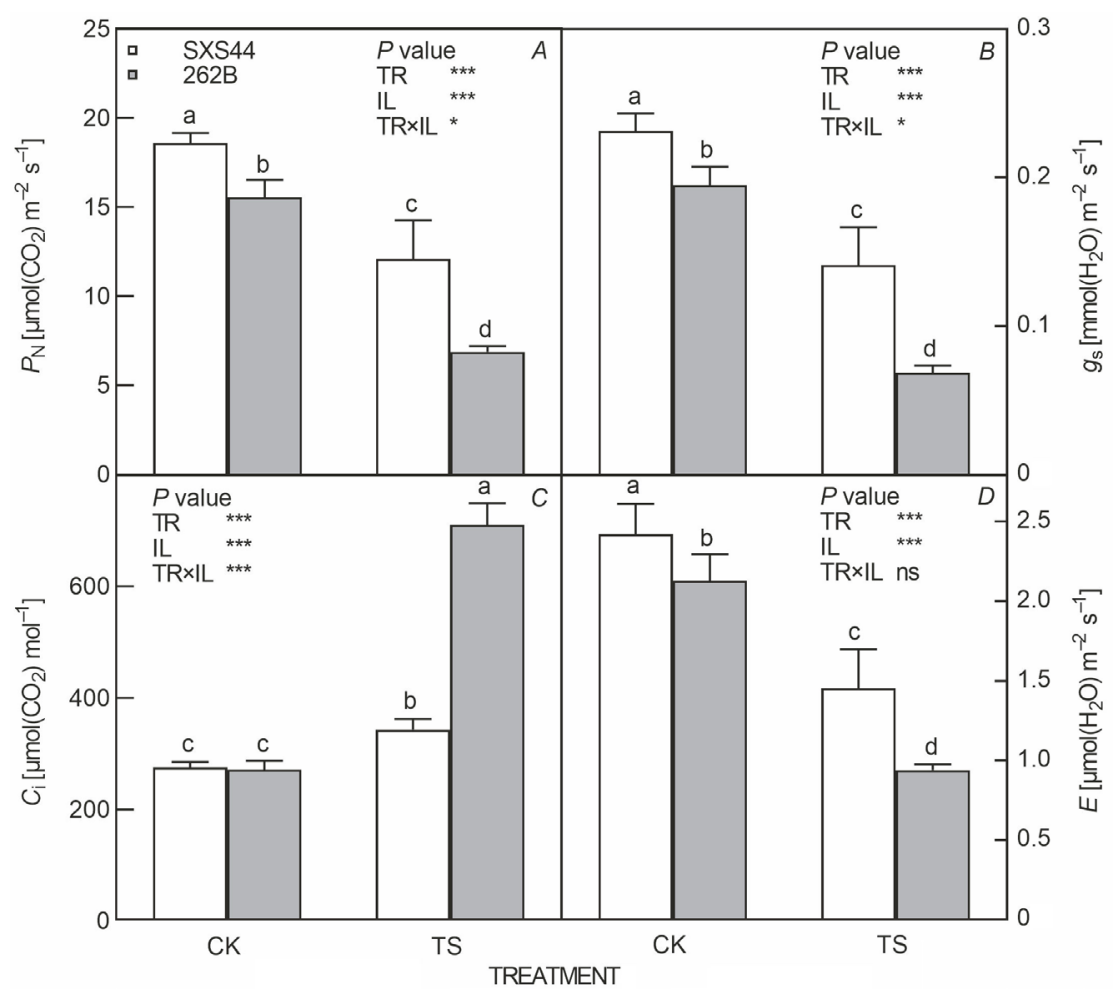

Fig. 5. Effect of alkaline stress on photosynthetic characteristics of sorghum seedlings. $(A)$ net photosynthetic rate $\left(P_{\mathrm{N}}\right) ;(B)$ stomatal conductance $\left(g_{\mathrm{s}}\right)$; $(C)$ intercellular $\mathrm{CO}_{2}$ concentration $\left(C_{\mathrm{i}}\right)$; $(D)$ transpiration rate $(E)$. TR - different treatments; IL - different inbred lines; CK - no alkaline stress; TS - alkaline stress. Significant differences $(P<0.05)$ were determined by different lowercase letters according to the one-way or two-way ANOVA followed by the least significant difference test. ${ }^{*}$ - significant at the $P=0.05$ level. $* *$ - significant at the $P=0.01$ level. *** - significant at the $P=0.001$ level. ns - no significant difference.

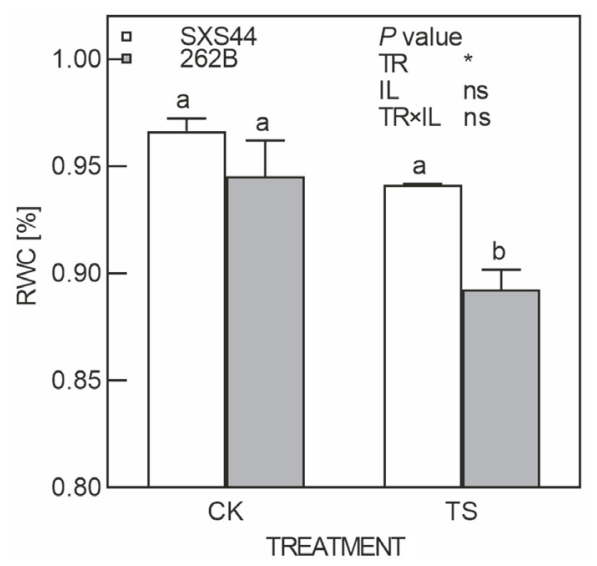

Fig. 6. Effect of alkaline stress on relative water content (RWC) of sorghum seedling leaves. TR - different treatments; IL - different inbred lines; CK - no alkaline stress; TS - alkaline stress. Significant differences $(P<0.05)$ were determined by different lowercase letters according to the one-way or two-way ANOVA followed by the least significant difference test. $*$ - significant at the $P=0.05$ level. ** - significant at the $P=0.01$ level. *** - significant at the $P=0.001$ level. ns - no significant difference.

The leaf water content of the SX44B and 262B showed significant positive correlation with $E\left(R^{2}=0.7623\right.$, $\left.p<0.05 ; R^{2}=0.6832, p<0.05\right)$. It indicated that in the presence of alkaline stress, the reduced leaf RWC had a negative effect on $E$ (Fig. $8 A, B$ ). There was an extremely significant negative correlation between $P_{\mathrm{N}}$ and $\Delta \mathrm{T}$

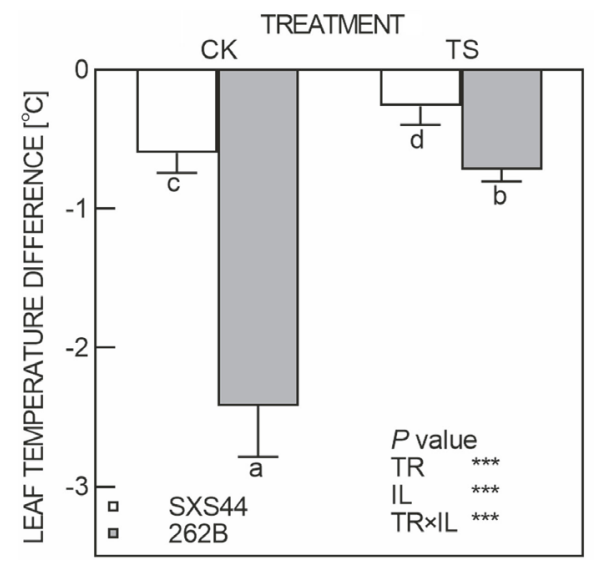

Fig. 7. Effect of alkaline stress on leaf temperature difference in sorghum seedlings. TR - different treatments; IL - different inbred lines; CK - no alkaline stress; TS - alkaline stress. Significant differences $(P<0.05)$ were determined by different lowercase letters according to the one-way or two-way ANOVA followed by the least significant difference test. $*$ - significant at the $P=0.05$ level. $* *$ - significant at the $P=0.01$ level. *** - significant at the $P=0.001$ level. ns - no significant difference.

$\left(R^{2}=0.6187, p<0.01 ; R^{2}=0.8893, p<0.01\right)$, which showed that higher $\Delta \mathrm{T}$ resulted in lower $P_{\mathrm{N}} . E$ was found to decrease with an increase in $\Delta \mathrm{T}$ (Fig. $8 C-F$ ). SX44B showed a significant negative correlation between $E$ and $\Delta \mathrm{T}\left(R^{2}=0.4853, p<0.05\right)$. 262B showed an extremely significant negative correlation between $E$ and $\Delta \mathrm{T}$ 

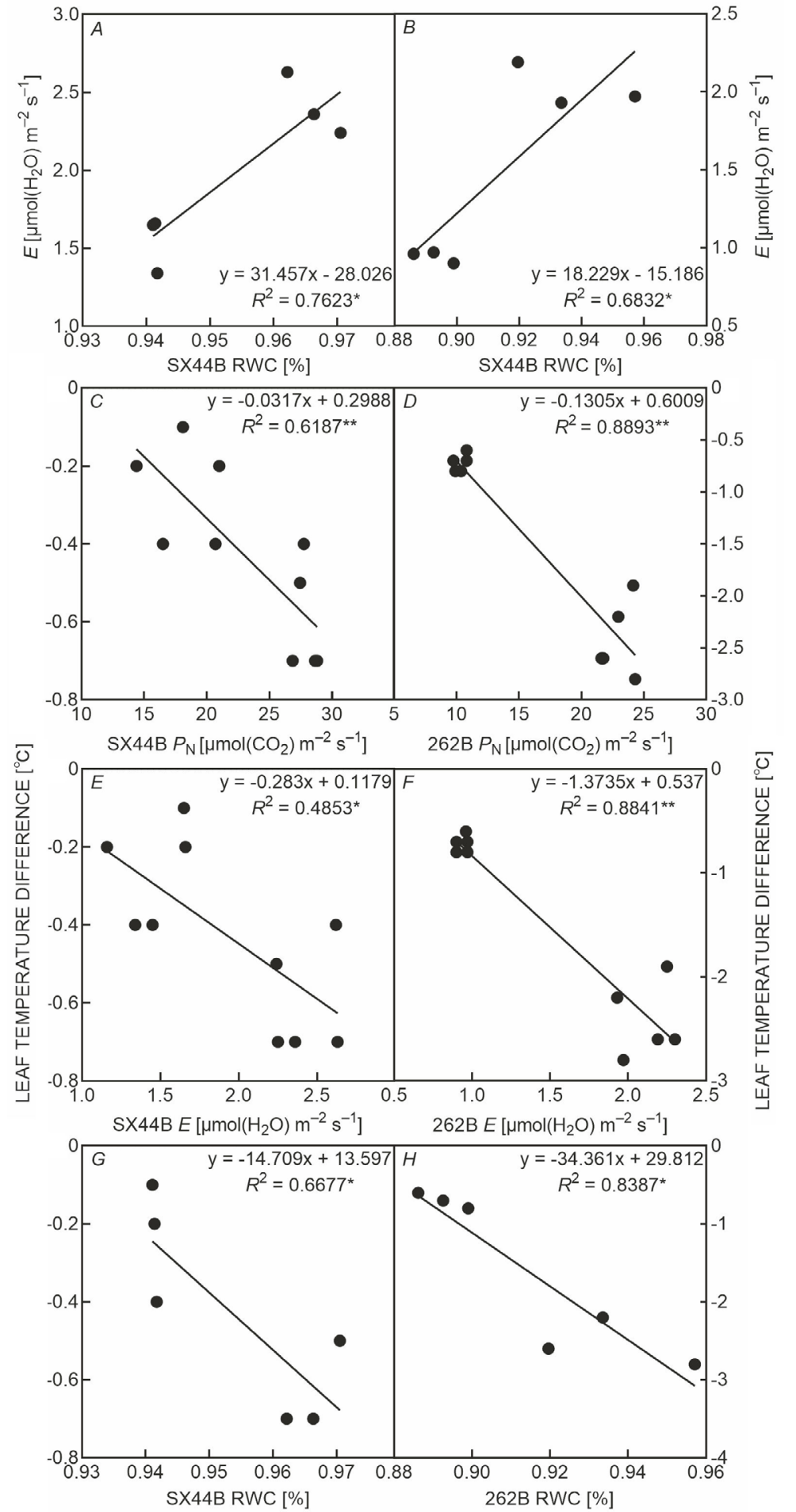

Fig. 8. Relationship between leaf RWC and $E$ $(A, B)$, leaf temperature difference and $P_{\mathrm{N}}(C, D)$, leaf temperature difference and $E(E, F)$, leaf temperature difference and leaf $\operatorname{RWC}(G, H)$ in sorghum seedlings under alkaline stress. $E-$ transpiration rate; RWC - relative water content; $P_{\mathrm{N}}-$ net photosynthetic rate; CK - no alkaline stress; TS - alkaline stress.
$\left(R^{2}=0.8841, p<0.01\right)$. Similarly, RWC and $\Delta \mathrm{T}$ also showed a significant negative correlation $\left(R^{2}=0.6677, p<0.05\right.$; $\left.R^{2}=0.8387, p<0.05\right)($ Fig. $8 G, H)$.

\section{Discussion}

Under alkaline stress, the morphological, physiological, and biochemical characteristics of crops change to adapt to the stress environment (Ge et al. 2010, Deinlein et al. 2014). In this experiment, the morphological characters of the two sorghum inbred lines were significantly different under conditions of mixed alkaline stress. After $3 \mathrm{~d}$ of stress, the leaves of 262B showed obvious withering and wilting, while the leaves of SX44B managed to maintain their shape, indicating SX44B showed better tolerance towards alkaline stress. The decrease of the fresh mass 
of SX44B under alkaline stress was lower than that of 262B, indicating that SX44B had better water retention ability and thus had better alkaline tolerance. There was no difference in dry matter accumulation between the two inbred lines under stress, which may be due to the short duration of stress treatment. The intracellular osmotic balance and ion balance are disrupted under alkaline stress (Guo et al. 2010). Therefore, the prompt elimination of excessive ROS is a standard to measure the alkaline tolerance of sorghum germplasm. Oxidative stress can damage cell structure and induce the production of ROS. A large amount of ROS can cause irreversible damage to chloroplasts, mitochondria, membrane lipids, and proteins, thereby damaging plant cells (Sivakumar et al. 2000, Affenzeller et al. 2009). In this experiment, the activities of SOD, POD, CAT, and APX in the leaves of the two sorghum inbred lines showed an upward trend under alkaline stress. It showed that alkaline stress can stimulate the activity of sorghum antioxidant enzymes to remove the ROS accumulated in the plants. However, the degree of enzyme activity was variable in different lines. Under alkaline stress, the activities of POD, CAT, and APX in SX44B leaves were significantly higher than that of 262B, especially POD and APX, indicating that SX44B had a stronger ROS-scavenging ability.

Chloroplasts are the main source of ROS, and excessive ROS leads to the damage of chloroplast structure (Ren et al. 2016, Ma et al. 2017, Zhou et al. 2018). Under alkaline stress, the chloroplast of $262 \mathrm{~B}$ deformed into a spherical shape. The double-membrane structure disappeared completely, and the thylakoid matrix lamella disintegrated and disappeared. When the thylakoid castellations reduce in size or even disappear, it reduces the absorption, transmission, and utilization of light energy, thus limiting the complete conversion of light energy into chemical energy and reducing photosynthetic capacity. By contrast, the chloroplast of SX44B still maintained a long oval shape. Some thylakoid basal granules were twisted and swollen, and the direction of arrangement was irregular. However, a part of the matrix sheet still maintained a good arrangement structure, and the double-layer membrane structure was also clearly visible. This may be because the alkali-tolerant variety SX44B maintained high antioxidant enzyme activity, resulting in less damage by ROS to the chloroplast. Ren et al. (2016) also found a similar phenomenon in maize. The change of chloroplast morphology and structure is an important factor affecting the Chl content under sorghum alkaline stress. As Chl is the main pigment of photosynthesis, its content also has an important effect on the photosynthesis of plants (Wang et al. 2018). In this experiment, alkaline stress treatment significantly reduced the Chl content of the seedlings of the two sorghum inbred lines. Chl content was significantly different between inbred lines and treatments, but not in interactions, suggesting that the higher Chl content may be the basis of resistance to the adverse environment such as alkaline stress. Furthermore, the net photosynthetic rate of alkali-sensitive variety 262B decreased more significantly. Additionally, the stomatal conductance and transpiration rate of sorghum decreased significantly, while the intercellular $\mathrm{CO}_{2}$ concentration increased significantly. This indicated that alkaline stress reduced the ability to assimilate $\mathrm{CO}_{2}$.

PSII plays an especially important role in photosynthesis response to environmental stresses (Baker 1991). Studies have found that alkaline stress can destroy the reaction center of PSII. The original light energy conversion efficiency $\left(\mathrm{F}_{\mathrm{v}} / \mathrm{F}_{\mathrm{m}}\right)$ is reduced, and light energy is excessive. At this time, it is necessary to convert light energy into heat and dissipate it to protect the photosynthetic system (Sun et al. 2012). $\mathrm{F}_{0}$ was related to the concentration of $\mathrm{Chl}$ in the leaves. In this experiment, $\mathrm{F}_{0}$ of SX44B remained higher under alkaline stress, indicating that the Chl concentration of SX44B could still maintain higher. The decrease in $F_{v} / F_{m}$ and $\Phi_{\text {PSII }}$ showed that the light energy conversion efficiency of sorghum was affected. Specifically, electron transfer was inhibited and photosynthetic activity was reduced. But it can also be found that the electron transfer of SX44B was less affected than 262B. NPQ is the nonphotochemical quenching coefficient. It reflects the ability of plants to convert light energy into heat when there is excessive light energy, which is the light-protection ability. Under alkaline stress, the increase in NPQ in SX44B revealed that alkaline stress affected the distribution of excitation energy in PSII of sorghum leaves. SX44B consumed excessive excitation energy through heat dissipation, which improved the lightprotection capability. To a certain extent, it alleviated the effect of alkaline stress on photosynthesis. The reduction of NPQ in 262B resulted in difficulty in consuming the excessive excitation energy accumulated in the plant over time, leading to damage to the photosynthetic system and a decrease in $P_{\mathrm{N}}$. The above results indicate that compared to the alkali-sensitive inbred line 262B, the alkali-tolerant inbred line SX44B can more effectively maintain the potential activity of PSII and the light energy conversion efficiency of PSII under alkaline stress. SX44B could effectively consume excessive light energy through heat dissipation. It protected the photosynthetic system and resulted in a decrease in the net photosynthetic rate. This may be a means by which SX44B protects PSII and resists alkaline stress.

Leaf water content affects the degree of stomatal opening and closing of crops (Ren et al. 2016). Closure of the stomata increases the resistance to $\mathrm{CO}_{2}$ exchange, which, in turn, reduces transpiration rate and restricts the prompt dissipation of heat in the leaves (Lu et al. 2003). When the water content of sorghum leaves decreases, their temperature increases (Wang et al. 2013). The change in leaf temperature can timely and accurately reflect the water content of leaves and the transpiration of plants (Zhang et al. 2019a). To avoid the influence of ambient temperature on leaf temperature, we used $\Delta \mathrm{T}$ to reflect the change in leaf temperature in this experiment. This experiment showed that under alkaline stress, the $\Delta \mathrm{T}$ of the two sorghum leaves increased significantly. Additionally, the increase of $\Delta \mathrm{T}$ in alkali-sensitive variety 262B was more obvious. Alkaline stress reduced the RWC of sorghum seedlings. Regression analysis showed a significant positive correlation between leaf water content 
and transpiration rate. It indicated that the decrease in leaf RWC under alkaline stress had a negative effect on $E$. There was a very significant negative correlation between $P_{\mathrm{N}}$ and $\Delta \mathrm{T}$. It revealed that the increase in leaf temperature can reflect the adverse effect of alkaline stress on $P_{\mathrm{N}}$. The $E$ of both SX44B and 262B had a significant negative correlation with $\Delta \mathrm{T}$. It illustrated that the leaf temperature of alkali-sensitive varieties responded more prominently to alkaline stress. Similarly, RWC and $\Delta \mathrm{T}$ also showed a significant negative correlation with each other. Therefore, we infer that the leaf water and photosynthetic physiology of sorghum under alkaline stress can be reflected by the change in $\Delta \mathrm{T}$. In other words, $\Delta \mathrm{T}$ can be used as a monitoring index to judge the degree of response of sorghum germplasm to alkaline stress.

Conclusion: Alkaline stress treatment destroyed the chloroplast structure and reduced $P_{\mathrm{N}}$. SX44B could eliminate the excessive ROS accumulated by alkaline stress and reduce the damage to the chloroplast by enhancing antioxidant enzyme activity. Moreover, it could keep a high NPQ and effectively consume excess light energy, thus maintaining a higher $P_{\mathrm{N}}$ and showing strong alkaline resistance. The temperature of sorghum leaves increased with the decrease of $E$ and $\Delta \mathrm{T}$ had a significant correlation with sorghum leaf water content and photosynthetic parameters. Thus, $\Delta \mathrm{T}$ can be used as an indicator to monitor the alkali tolerance of sorghum. This study provides an effective method for future alkalitolerant genotype testing.

\section{References}

Affenzeller M.J., Darehshouri A., Andosch A. et al.: Salt stressinduced cell death in the unicellular green alga Micrasterias denticulata. - J. Exp. Bot. 60: 939-954, 2009.

Amini S., Ghadiri H., Chen C.R., Marschner P.: Salt-affected soils, reclamation, carbon dynamics, and biochar: a review. J. Soil. Sediment. 16: 939-953, 2016

Baker N.R.: A possible role for photosystem II in environmental perturbations of photosynthesis. - Physiol. Plantarum 81: 563-570, 1991

Blum A., Mayer J., Gozlan G.: Infrared thermal sensing of plant canopies as a screening technique for dehydration avoidance in wheat. - Field Crop. Res. 5: 137-146, 1982.

Choudhury F.K., Rivero R.M., Blumwald E., Mittler R.: Reactive oxygen species, abiotic stress and stress combination. - Plant J. 90: 856-867, 2017.

Deinlein U., Stephan A.B., Horie T. et al:: Plant salt-tolerance mechanisms, - Trends Plant Sci. 19: 371-379, 2014.

Fielding J.L., Hall J.L.: A biochemical and cytochemical study of peroxidase activity in roots of Pisum sativum: I. A comparison of DAB-peroxidase and guaiacol-peroxidase with particular emphasis on the properties of cell wall activity. - J. Exp. Bot. 29: 969-981, 1978

Ge Y., Li Y., Zhu Y.M. et al.: Global transcriptome profiling of wild soybean (Glycine soja) roots under $\mathrm{NaHCO}_{3}$ treatment. BMC Plant Biol. 10: 153, 2010.

Guo L.Q., Wang H.Y., Ma Y. et al.: [Mechanisms of osmotic adjustment and ionic balance in Puccinellia tenuiflora in response to salt and alkali stresses.] - J. Northeast Norm. Univ. 42: 120-125, 2010. [In Chinese]

Huang R.D.: Research progress on plant tolerance to soil salinity and alkalinity in sorghum. - J. Integr. Agr. 17: 739-746, 2018.

Hussain S., Iqbal N., Brestic M. et al.: Changes in morphology, chlorophyll fluorescence performance and Rubisco activity of soybean in response to foliar application of ionic titanium under normal light and shade environment. - Sci. Total Environ. 658: 626-637, 2019.

Leng C.X., Zheng F.Y., Zhao B.P. et al.: [Advances on alkaline tolerance of rice.] - Biotechnol. Bull. 36: 103-111, 2020. [In Chinese]

Liu X.: [Research on the limitation of stomatal and non-stomatal to ban photosynthesis under the $\mathrm{Na}_{2} \mathrm{CO}_{3}$ stress.] - J. Anhui Agric. Sci. 38: 2861-2862+2913, 2010. [In Chinese]

Lu C.M., Qiu N.W., Wang B.S., Zhang J.H.: Salinity treatment shows no effects on photosystem II photochemistry, but increases the resistance of photosystem II to heat stress in halophyte Suaeda salsa. - J. Exp. Bot. 54: 851-860, 2003.

Ma J., Lv C.F., Zhang B.B. et al.: Comparative analysis of ultrastructure, antioxidant enzyme activities, and photosynthetic performance in rice mutant $812 \mathrm{HS}$ prone to photooxidation. - Photosynthetica 55: 568-578, 2017.

Munns R., Tester M.: Mechanisms of salinity tolerance. - Annu. Rev. Plant Biol. 59: 651-681, 2008.

Noreen S., Ashraf M., Hussain M., Jamil A.: Exogenous application of salicylic acid enhances antioxidative capacity in salt-stressed sunflower (Helianthus annuus L.) plants. Pak. J. Bot. 41: 473-479, 2009.

Qiao X.Q., Shi G.X., Chen L. et al.: Lead-induced oxidative damage in steriled seedlings of Nymphoides peltatum. Environ. Sci. Pollut. R. 20: 5047-5055, 2013.

Ren B., Zhang J., Dong S. et al.: Effects of duration of waterlogging at different growth stages on grain growth of summer maize (Zea mavs L.) under field conditions. J. Agron. Crop Sci. 202: 564-575, 2016.

Ren Z.G., Gao Z.Y., Zhang Y. et al.: [The influence of mixed salt and alkali stress on physiological and agronomic traits of sorghum.] - Crops 1:100-106, 2017. [In Chinese]

Sewelam N., Kazan K., Schenk P.M.: Global plant stress signaling: Reactive oxygen species at the cross-road. - Front. Plant Sci. 7: 187, 2016.

Sivakumar P., Sharmila P., Saradhi P.P.: Proline alleviates saltstress-induced enhancement in ribulose-1,5-bisphosphate oxygenase activity. - Biochem. Bioph. Res. Co. 279: 512515,2000

Sun W., Ubierna N., Ma J.Y., Cousins A.B.: The influence of light quality on $\mathrm{C}_{4}$ photosynthesis under steady-state conditions in Zea mays and Miscanthus $\times$ giganteus: changes in rates of photosynthesis but not the efficiency of the $\mathrm{CO}_{2}$ concentrating mechanism. - Plant Cell Environ. 35: 982-993, 2012.

Tuna A.L., Kaya C., Dikilitas M., Higgs D.: The combined effects of gibberellic acid and salinity on some antioxidant enzyme activities, plant growth parameters and nutritional status in maize plants. - Environ. Exp. Bot. 62: 1-9, 2008

Wang Y.N., Jie W.G., Peng X.Y. et al.: Physiological adaptive strategies of oil seed crop Ricinus communis early seedlings (cotyledon vs. true leaf) under salt and alkali stresses: from the growth, photosynthesis and chlorophyll fluorescence. Front. Plant Sci. 9: 1939, 2018.

Wang Y.T., Zhou Y.F., Li F.X. et al.: [Relationship between leaf temperature and water status in sorghum under drought stress.] - Agr. Res. Arid Areas 31: 146-151, 2013. [In Chinese]

Wang Z.C., Yang F., Qi C.Y., Liang Z.W.: [Osmotic regulation response of rice to soil salinity and alkalinity stresses.] - Agr. Res. Arid Areas 28: 153-157, 2010. [In Chinese]

Wintermans J., De Mots A.: Spectrophotometric characteristics of chlorophylls $a$ and $b$ and their pheophytins in ethanol. BBA-Biophysics 2: 448-453, 1965. 
Yang C.W., Xu H.H., Wang L.L. et al.: Comparative effects of salt-stress and alkali-stress on the growth, photosynthesis, solute accumulation, and ion balance of barley plants. Photosynthetica 47: 79-86, 2009.

Yang J.Y., Zheng W., Tian Y. et al.: Effects of various mixed salt-alkaline stresses on growth, photosynthesis, and photosynthetic pigment concentrations of Medicago ruthenica seedlings. - Photosynthetica 49: 275-284, 2011.

Yin D., Chen S., Chen F. et al.: Morphological and physiological responses of two chrysanthemum cultivars differing in their tolerance to waterlogging. - Environ. Exp. Bot. 67: 87-93, 2009.

Yordanova R., Christov K.N., Popova L.P.: Antioxidative enzymes in barley plants subjected to soil flooding. - Environ. Exp. Bot. 51: 93-101, 2004.

Zhang R.D., Zhou Y.F., Yue Z.X. et al.: Changes in photosynthesis, chloroplast ultrastructure, and antioxidant metabolism in leaves of sorghum under waterlogging stress. Photosynthetica 57: 1076-1083, 2019b.

Zhang R.D., Zhou Y.F., Yue Z.X. et al.: The leaf-air temperature difference reflects the variation in water status and photosynthesis of sorghum under waterlogged conditions. PLoS ONE 14: e0219209, 2019a.

Zhao Q., Suo J.W., Chen S.X. et al.: $\mathrm{Na}_{2} \mathrm{CO}_{3}$-responsive mechanisms in halophyte Puccinellia tenuiflora roots revealed by physiological and proteomic analyses. - Sci. Rep.-UK 6: 32717, 2016.

Zhou W.Y., Chen J.H., Lu L. et al.: [Changes on leaf chloroplast ultrastructure and photosynthetic characteristics of Liriodendron sino-americanum somatic embryo regeneration seedlings under waterlogging stress.] - Sci. Silv. Sin. 54: 1928, 2018. [In Chinese]

(C) The authors. This is an open access article distributed under the terms of the Creative Commons BY-NC-ND Licence. 